\title{
An expert system for selecting attribute sampling plans
}

\author{
NASSER S. FARD and IHSAN SABUNCUOGLU
}

\begin{abstract}
A considerable portion of quality control managers' time in a production system is spent in routine and complex decision-making processes that have significant impact on average outgoing quality, quality improvement, and quality cost. The quality control manager must decide among various statistical process control methods and sampling plans for each part (or characteristic). These decisions are usually based on how critical a part is, historical information about the quality of the parts, and other factors. Many of these factors require subjective judgments by the quality control manager. For a production facility with an inventory system of thousands of different parts, determination of feasible sampling plans and process control charts is a time-consuming and difficult task. As demonstrated in this paper, an expert system has been designed to facilitate the selection of an appropriate sampling plan for cach part. The system is referred to as Adviser for Selecting Acribute Sampling Plan (ASASP).
\end{abstract}

\section{Introduction}

Quality control is a process of detecting nonconforming parts (those not meeting standards defined by the producer and consumer) in production lots that contain both conforming and non-conforming products. The results obtained from the quality control will show the variation in a process, and help to determine the cause(s) of failure(s). Corrective actions may then be taken to remove the source of a particular problem.

Statistical quality control applies appropriate control chart, sampling plan, and other statistical methods to determine whether the quality of an item or a product under investigation satisfies a set of specifications defined by management, engineers, and customers. The quality control manager must choose feasible control charts and an appropriate sampling plan for a particular product to decide about the acceptability of that product. Very often these decisions do not come easily; there are many res-

Authors: Nasser S. Fard, Department of Industrial Engineering and Information Systems, Northeastern University, Boston, MA 02115 , USA; and Ihsan Sabuncuoglu, Department of Industrial Engineering, Bilkent University, Ankara, Turkey 06572. trictions, such as lack of equipment, knowledgeable personnel, costs, historical data, and quantity of available data which may prevent one from choosing the most appropriate method.

A successful quality control programme involves all production levels and all product lines. The first step in creating a programme that will be able to meet the goals and objectives of any system is sound quality planning. A poor quality planning programme which fails to set up feasible goals and standards, and eliminate the source of troubles, leads to inherent wastes at the operating level. Waste reduction does not happen of its own accord; it results from purposeful action taken by upper management through a quality improvement process (Juran 1986).

Sampling plans are statistical methods, designed to determine sample size and decision criterion for production lots with different size and quality. Application of an appropriate sampling plan will result in sufficient and necessary sample size to estimate a lot quality, which will lead to acceptance or rejection of that lot (Duncan 1974, Schilling 1985).

In a typical manufacturing environment, where hundreds of different parts and units are received and produced every day, with various characteristics and specifications, a large number of decisions at various stages of production processes are required. There are many variables that require a continuous review to assure that the standards and criteria are satisfied. These include raw material, machine set-up, the supplier quality, and precision of tools and equipments. Design and application of a sampling plan for each part, material, or characteristic in this environment is not an easy task. The Adviser for Selecting Attribute Sampling Plan (ASASP) is designed to assist the user in selection and application of a most appropriate sampling plan for a particular part or characteristic.

The second section of this paper presents the importance of application of the expert system (ES) in quality control. After a brief summary of ES and its applications, section 3 focuses on acceptance sampling plans and their 
parameters. Details of the prototype ES are given in section 4 , and this is followed by a summary and concluding remarks.

\section{Expert systems}

Advancements in information system technology, and particularly in the area of ES, allow for representations of experienced expert knowledge within the structure of these systems.

In recent years, ES has been applied successfully in many areas that require decision-making, such as manufacturing, optimization, and medical diagnosis. Technological advancement in manufacturing, and use of complex robotics have shown the need for AI and ES (Sabuncuoglu and Hommertzheim 1989). In computerintegrated manufacturing (CIM) systems, where computers control most manufacturing activities with a minimum degree of human interruption, various ES have been developed (Hosseini and Fard 1989, Dagli and Stacey 1988, Kusiak and Chen 1988, De et al. 1985).

A decision-maker utilizes the advice of an expert in a complex decision-making environment to arrive at solution. Human experts can achieve high performance in a specific task if they are knowledgeable about that task. If this knowledge is transferred into computer programs, such that the computer becomes capable of analysing data, evaluating the rules, and achieving high performance in the role of decision-making, then a significant amount of the human expert time can be saved, and it can be utilized in a number of different tasks.

Quality control is an important part of manufacturing and production control. There are a variety of quality control methods, each requiring special information. ES is a desirable tool to facilitate the decision-making process (by a quality control manager) in selection of appropriate control charts, decision to choose an appropriate sampling plan, or quality cost analysis. For example, many quality control decisions in the CIM environment require expertise to determine appropriate methods for data collection, analysis, and decisionmaking. Through an ES the human expertise about a particular task may be combined with required conditions, assumptions, and criteria of various models to choose a most feasible model for a particular application. This may consist of selecting an appropriate sampling plan or a feasible control chart that will provide a powerful and objective tool for the quality control manager to accept or reject a given production lot. This approach will not only reduce the quality control manager's time in routine decision-making, but the data analysis time and the decision-making time itself will also be reduced.

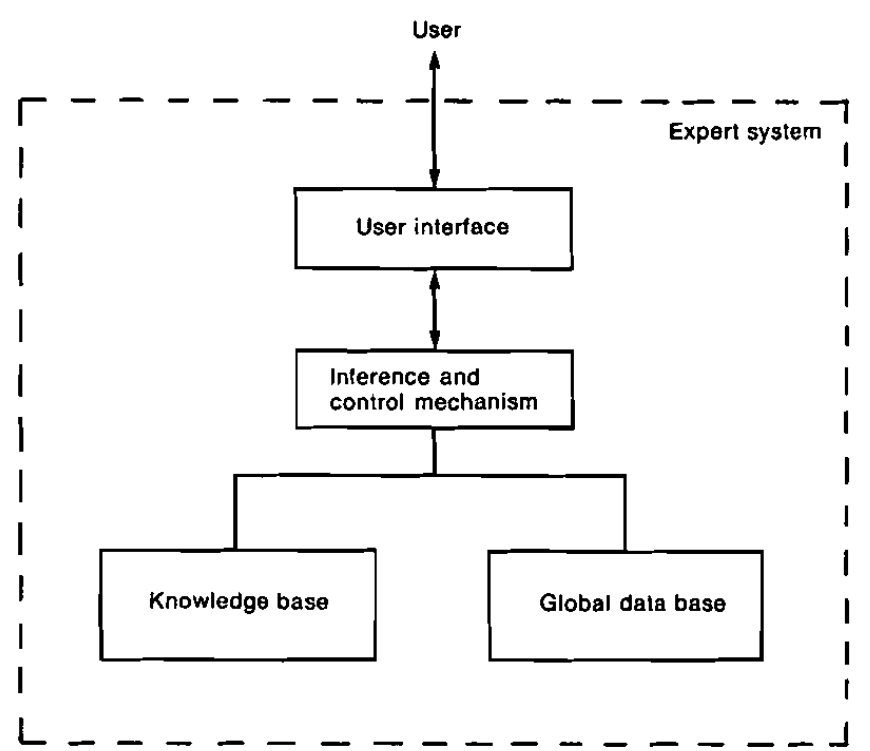

Figure 1. A schematic view of an ES.

In general, there are three primary elements of an ES (Figure 1). A knowledge base, the kernel of the ES, consists of facts and heuristic knowledge about the problem domain. It is usually procedural, and defines the method of problem-solving given the available current information. A global data base stores necessary information, and it keeps track of the stages in the problem solving process. As compared to the knowledge base, the global data base is descriptive. An inference engine, however, is a control mechanism of the ES which utilizes the knowledge base and the global data base to arrive at conclusions or recommendations to a user. Knowledge and expertise related to a specific problem domain can be easily added and deleted without affecting control mechanisms or other elements of an ES. A user interface can be provided in various forms: textual (natural language); numerical (detail); or graphical.

An ES uses heuristic search methods and employs symbolic processing in contrast to conventional optimization techniques which are basically numeric and use algorithmic search methods. This characteristic makes ES very powerful when dealing with complex and illstructured problems that involve many qualitative as well as quantitative factors, and require fast response. For a detailed information about the expert systems see Waterman (1986) and Hayes-Roth et al. (1983). There are also similarities between ES and conventional optimization methods (O'Keefe 1985). There is a growing tendency in the literature to combine an ES and classical optimization techniques to increase the robustness, efficiency, and quality of models and solutions for multi-product manufacturing, or service industries. This combined approach (which is called ES in tandem mode) 
was proposed for the scheduling of automated manufacturing systems (Kusiak 1987).

A brief description of acceptance sampling plans with various factors involved in analysis and selection of a feasible sampling plan is discussed in the next section. Applications, restrictions, and parameters of sampling plans arc also presented.

\section{Selecting the appropriate acceptance sampling plan}

Acceptance sampling plans are frequently used in manufacturing environments, to determine the sample size and decision criterion for acceptance (or rejection) of a lot. The acceptance criterion and sample size are determined based on a set of factors such as the existing fraction defective in a process, lot tolerance fraction defective, and maximum allowable risk levels (type I risk-probability of rejecting conforming part; and type II risk-probability of accepting nonconforming part). The risk levels are usually determined based on agreements between producer and consumer. Lower risk levels will lead to higher sample size for inspection which results in higher cost. A careful study of process fraction defective, inspection cost, as well as criticality of quality of a particular part or product by both producer and consumer are done choosing feasible risk levels.

In general, acceptance sampling plans may be divided into four categories (Dodge 1969):

(i) lot-by-lot attribute sampling, in which units in a sample are inspected on a go-not-go basis for one or more characteristics;

(ii) lot-by-lot variable sampling, in which units in a sample are measured for a single characteristic;

(iii) continuous sampling of a flow of units by attributes;

(iv) special purpose sampling including chain sampling and skip-lot-sampling

Of the first two categories the lot-by-lot attribute sampling plans have been preferred in most circumstances, since

(i) variable sampling plans require separate charts for each quality characteristic, while attribute sampling plans may be designed for investigating units with one or more quality characteristics;

(ii) using the variable sampling plan, lot may be rejected without detection of a non-conforming unit. In attribute sampling, at least one nonconforming unit is required to reject a lot.

(iii) variable sampling plans usually assume a normal distribution for a measurable characteristic under investigation; an attribute sampling plan, however, assumes a distribution based on the nature of the problem, sample size, and available information;

(iv) computational procedures to obtain the required statistics and sample size are lengthier in variable sampling plans than they are in attribute sampling.

Selection of an appropriate attribute sampling plan for a particular part or product should consider many factors such as a process fraction defective, inspection cost, type, and size of available information about the part (such as lot size and average sample size). Comparison of a double and a single sampling plan shows (Fard 1985) that for a large and small average quality level (AQL), the average sample size is less using the double sampling plan (Fig. 2). A typical organization deals with a large quantity of parts and finished products, and the decision to choose appropriate sampling plan for all parts or characteristics is a complex and time consuming task. Today, the situation is further complicated by the fact that corporations have to operate in a highly dynamic marketing and manufacturing environment. Therefore, selecting a sampling plan for a particular item is a continuous decision process which requires on-going update and revision as a result of changes in parameters' values such as cost, product-mix and managerial planning policies, etc. A desirable sampling plan designed for a particular product may not be feasible for other products or different suppliers. Therefore, there is a real need to design a practical expert system in which quality planning and quality control decisions could be made more efficiently in a real time. The emergence of CIM systems especially accelerates this need.

The selection of an attribute sampling plan is a complex decision involving number of subjective factors.

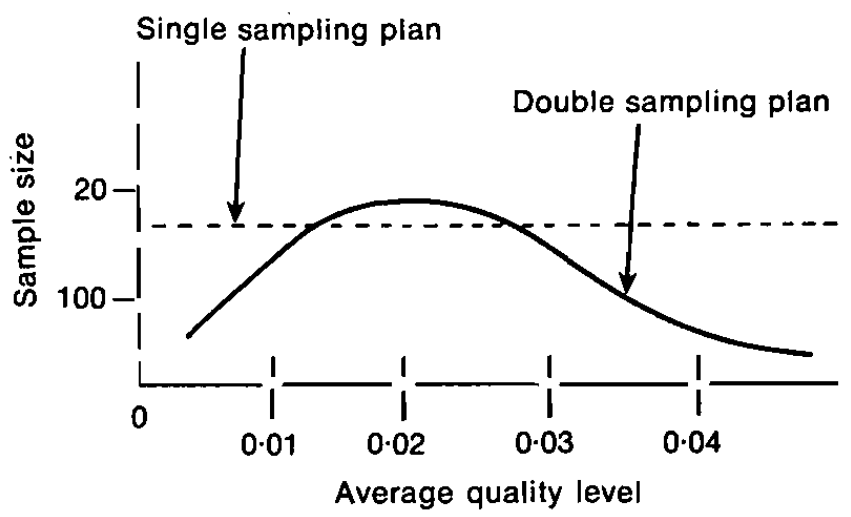

Figure 2. Average sample size for single and double sampling plans. 
In addition to the predetermined levels of type I and type II risks, lot fraction defective, inspection cost, type of inspection (destructive or non destructive inspection), acceptable level of average outgoing quality, and lot size are among the factors affecting the selection of sampling plan. Since the total amount of sample size in some attribute sampling plans is not known prior to inspection and the inspection result of one sample will determine the need for further samples, flexibility of inspection time is also another subjective input data provided by the user. Given a set of information about product quality, production system, and lot, ASASP selects a sampling plan for that product.

In some circumstances the election of an appropriate sampling plan is trivial. For instance, if the acceptable number of non-conforming parts is restricted to zero, then selection of a single sampling plan becomes inevitable. Factors which influence the selection procedure are either quantitative or qualitative. The quantitative factors include inspection cost (i.e., fixed cost, variable cost), sample size number of defective items, producer risk, and consumer risk. Their values are based on objective evidence resulting from theoretical models and empirical data. These parameters can be measured based on historical data, accounting records, engineering design specification, and customer requirements. On the other hand, qualitative factors can not be easily measured. Their values are usually determined based on on subjective beliefs and past experiences. Some of the qualitative factors surrounding acceptance sampling problems are listed below:

- acceptability of a particular sampling plan by the producer;

- difficulty of using a particular sampling plan (i.e., teaching line inspectors, variability of inspection load, inspector's errors, etc.);

- relative importance of each quantitative and qualitative factor (i.e., impact of the damage as a result of the use of non-conforming parts);

- complexity of application;

- other management strategies and operational policies, etc.

All these factors must be considered in the analysis and selection of the most feasible sampling plan for a particular product.

\section{The ASASP System}

An ES can be characterized along the following dimensions: the problem domain; representation of knowledge (i.e., structure of the knowledge base); the inference mechanism; and the user interface.
Selection of an appropriate sampling plan is the domain of the ES. Due to their wide application in manufacturing and service systems, the current ES choices were restricted to three types of attribute sampling plans, namely single sampling plan, double sampling plan, and multiple sampling plan.

The ASASP described in this paper considers many essential quantitative factors such as lot fraction defective and inspection cost, and number of qualitative factors such as flexibility of inspection time and level of the user's knowledge about various sampling plans.

Selection of a knowledge-base representation scheme and inference mechanism is an important issue in ES research (Waterman 1986). Use of an appropriate language (i.e., PROLOG, LISP, C, etc.) and selection of particular hardware configuration is another factor which must be considered in the early stages of an ES development. The entire process is highly dependent on the nature of the problem at hand (i.e., planning, diagnosing, etc.), the designer's experience, and his or her familiarity with existing languages and tools. Today, there are also a number of general purpose ES development tools which facilitate the development of ES for a particular application.

The ASASP considered in this study demonstrates an application of ES in a complex decision making environment involving many factors. Because the knowledge in ASASP could be represented using rules, a rule based knowledge representation scheme was chosen. Use of backward chaining strategy was employed since the possible outcomes are usually known to the users. It is efficient in terms of problem solving. Therefore, the expert system development tool M.1 (1985) is used in developing the ES. Rule-based knowledge representation and the backward-chaining inference mechanism are appropriate methods for this diagnosis/prescription paradigm. The M.1 allows both alternatives. In addition, the knowledge engineer was familiar with this system and M.1 was available to be used for developing ASASP.

The M.1 mainly consists of a knowledge base, inference engine, working memory (global data base), and a user's input/output interface. The knowledge base is developed in the form of rules by using any standard text editor such as Wordstar. Execution of rules and facts is controlled by backward chaining. The resulting ES runs in the DOS environment on any IBM PC/XT/AT or PC compatible computer. Some details of the ES are given in the next subsection.

\subsection{Structure of knowledge base}

As depicted in Fig. 3, the knowledge-base consists of rules and facts. The rules can be viewed as 'if/then state- 


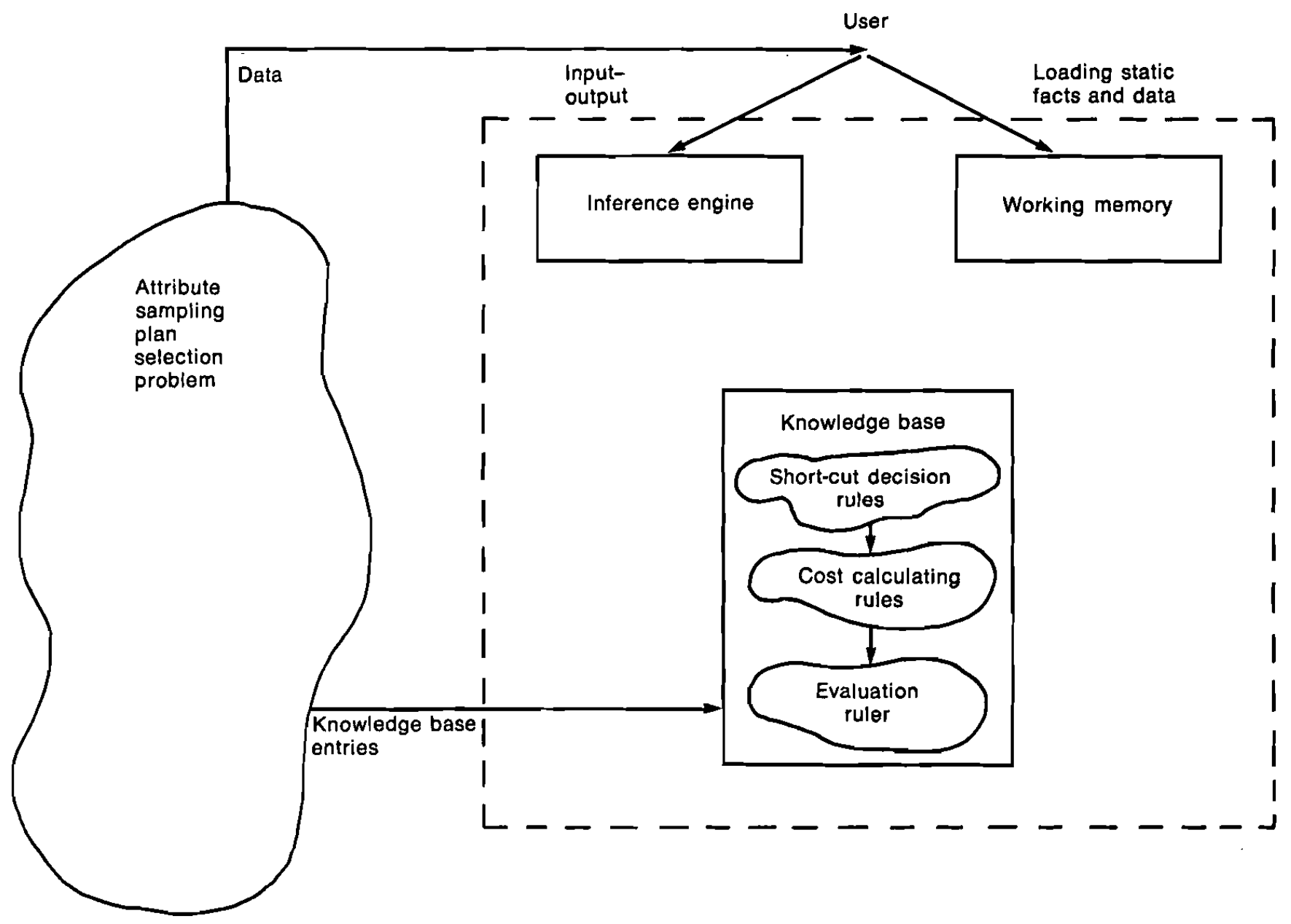

Figure 3. A schematic view of the proposed ES selecting the sampling plan.

ments. Each rule has a condition part ('if' portion) and an action apart ('then' portion). The true value of the 'if' portion is checked based on the facts and the previous conclusions, which are stored in the working memory (global data base). Whenever the left side of the rule (or prenise) is true, then the right side of the rule is cxccuted. Changes are made in the working memory based on new conclusions. A few sample rules are given in Table 1.

The domain expert and knowledge engineer worked closely together to develop a formalized knowledge structurc. The domain except specialization is in quality control and applied statistics. The knowledge engineer played a key role in the development of the system. The knowledge acquisition process was through interview, problem discussion and analysis. For example, the domain expert provided all factors which could be involved in selection of the double sampling plan, such as: lot fraction defective, lot size, cost of inspecting an itcm, feasible inspection time per lot, level of users' knowledge about different sampling plans, and criticality of the remaining fraction defective in uninspected portion of a lot.

The facts are the pieces of evidence or information that are necessary to arrive at conclusions when solving a.particular problem. They can be obtained either directly from the working memory or from the user by asking questions during the consultation. All of the intermediate conclusions via the rules are also considered to be facts. For example, if the value of 'least favourable' equals 2, then this simple evidence can be represented as:

$\mathrm{kb}-36$ : factor (least favourable) $=2$.

Moreover, in ES terminology, the facts which are obtained from the user in the form of 'question statements' can also be called 'meta-facts. For example, during the consultation, if the value of the expression regarding the producer's acceptance of double sampling is not know, then the control mechanism executes the following meta-fact:

$\mathrm{kb}-56$ : question (double-acceptability) $=[\mathrm{n} 1$, 'what do you think about the acceptability of double sampling plan?'] 
Table 1. Sample rule.

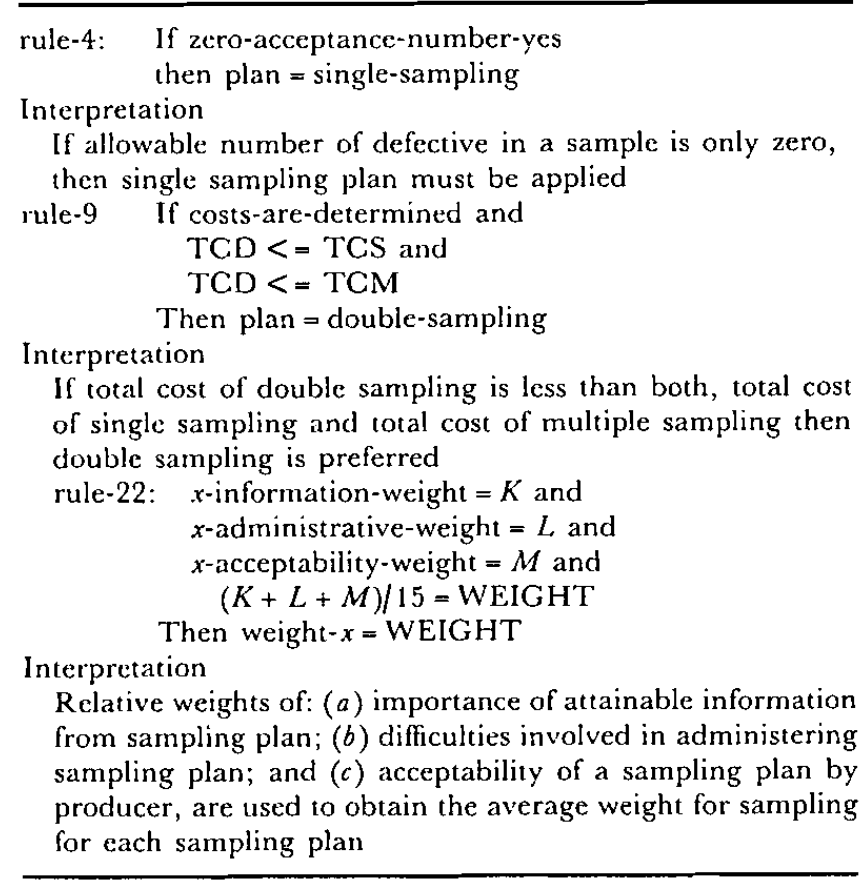

The ASASP inference engine employs backward chaining with a 'depth-first search'. Since the resulting ES is a 'goal-driven' system, it takes every opportunity to produce subgoals, and from these subgoals to achieve the desired goal (i.e., selecting an appropriate sampling plan).

In the current ASASP, in order to increase programming efficiency and to reduce the consultation time, the rules are organized into the following hierarchical groups;

(i) Short-cut decision rules: These are the rules which recommend a solution (i.e., sampling plan) without searching the knowledge-base further. They represent the decisions for trivial cases. For example, if a sample is taken under conveyorized production, or the acceptable number of nonconforming parts is zero, then the single sampling plan is recommended.

(ii) Cost-calculating procedural rules: These are the rules which are invoked if short-cut decision rules were unsuccessful. They collect information about costs, sample size, number of defective, probability of acceptance, etc., and then calculate the inspection cost for each alternative sampling plan. At this stage, the control mechanism first searches the working memory (global data base). If the facts related to the these parameters are not available in the data-base, then the user is informed to provide values for these parameters. (ii) Evaluation rules: These are the rules used to determine the weight of each qualitative factor, and to adjust the inspection cost so that the alternative plan having the least penalty is selected.

In case of incomplete information, the system is capable of taking default values to arrive at a conclusion. The user interface is also simple: all consultation takes place in a 'question/answer' manner and inputs can be either numeric or in limited textual form (i.e., verbal), in which the user can respond to the questions in 'English-like' statements. Because M.1 has 'explanation' facilities, the resulting ES is capable of showing current conclusions with associated reasoning at each stage of consultation.

\subsection{Working memory}

The working memory is one of the important elements of the current ES. It acts as a global data base which stores all the data and information about both intermediate and final conclusions. In many respects, it is useful to divide the global data base into the following elements:

(a) static data base;

(b) dynamic data base.

The static data base stores permanent information and does not change with time. On the other hand, the dynamic data base is the source of information which change with time. In manufacturing applications of ES (Ben-Arieh 1986), the static data base stores the job types, processing sequence, and layout of machines, all of which usually do not change so often. However, the status of machines, the queue sizes, etc. are stored in the dynamic data base. In the designed ASASP, some parameters of an acceptance sampling plan such as probability of accepting maximum number of allowable non-conforming units in a lot, and sampling cost can be categorized as the static if their values do not change frequently. Other parameters can be treated as the dynamic data base. Separation of the static and dynamic elements in the global data base helps to reduce the computational time inherent in the existing ES. In the current prototype ES, there is no physical separation of dynamic and static data bases. However, M.1 allows for saving and loading the information from a disk file. This feature makes it possible to load a set of facts (or static data) without asking the user to input them one by one during a consultation. This disk file may be either the output of a previous consultation or created by some external program. Therefore, in the current ASASP, it is the user's choice to decide static elements based on his/her particular application and provide this set of facts. 
In contrist to classical systems, there are significant difliculties in evaluation of ES. First, it is extremely dificult to evaluate the expertise, since the human cxperts are rarely evaluated objectively. Second, it is not clear yet, whether ES should be evaluated against the expert, or some evaluation criteria in the domain of the problem, or both. Third, it is very difficult to find some objective criteria against expert, where ES can be evaluated. The evaluation process of ASASP was done through the sample test cases. Also evaluation of the ES should be continuous process; as the feedback are oblained from various expert users.

A sample consultation is given in appendix to demonstratc the execution of the ASASP.

\section{Conclusion}

This study presented an ES approach to assist the quality engineer in selecting an appropriate sampling for his/her application. Selection and application of sampling plan for cach part/characteristic is a complex and time consuming process, requiring knowledgeable and trained personnel. For a production process consisting of large quantity of products with different values, quality and standards, the selection process becomes even more complicated.

The developed ASASP is based on the idea of integrating quantitative methods with artificial intelligence techniques and concepts. We believe that today's problcms, especially the manufacturing problems in the CIM environment, are highly complicated and can be handled by using a combination of approaches such as expert systems and quantitative optimization techniques.

\section{References}

BArR, A., and Fieicinnaum, E., 1981, The Handbook of Artificial Intelligence, Volume 1 (Stanford, CA: Heuristech Press).

BEN-ARIEH, D., 1986, A knowledge-based system for simulation and control of FMS, in Simulation Applications in Manufacturing ed. Hurrion (IFS, London), 287-295.

DACla, C. H., and STACEY, R., 1988, A prototype expert system for selecting control charts, International Journal of Production Research, 26, 987-996.

Dagli C., and SABuncuoglu, I., 1989, A prototype expert system for selecting MRP lot sizing heuristics based on fuzzy demand pattern, in Fuzzy Methodologies for Industrial and Systems Engineering, ed. G. V. Evans, W. Karwroski, and M. R. Wilhelm (Elsevier, Amsterdam), 299-309.

Di, S., NOF, S. Y., and WhINSTON, A. B., 1985, Decision support in computer-integrated manufacturing, Decision Support Systems, 1, 37-56.

Dolsct, H. F., 1969, Notes on the evaluation of acceptance sampling plans, Part I, Journal of Quality Technology, 1, $77-88$.

Duncan, A. J., 1974, Quality Control and Industrial Statistics, (Richard D. Irwin, Inc., Homewood, IL).

FARD, N. S., 1985, Software design for single-sample fractiondefective plan, Proceeding of Computers and Industrial Engineering, 9, 262-264.

Harmon, P. and King D., 1985, Expert Systems (Wiley, New York).

Hayes-roth, F., Waterman, D., and Lenat, D., 1983, Building Expert Systems (Addison-Wesley, Reading, MA)

HOSSEINI, J. and FARD, N. S, 1989, Conceptualization and formulization of knowledge in a knowledge based quality assurance system, Proceedings of the International Conference on Expert Systems and the Leading Edge in Production Planning and Control, Hilton Head, South Carolina.

JURAN, J. M., 1986, Universal approach to managing for quality: the quality trilogy, Quality Progress, XIX, number 8 $19-24$.

KUSIAK, A., 1987, Designing expert system for scheduling of automated manufacturing, Industrial Engineering, 19, 42-46.

KusiaK, A. and Chen, M., 1988, Expert systems for planning and scheduling manufacturing systems, European Journal of Operational Research, 34, 113-130.

M.1 Reference Manual for Software Version 2.1, 1985 (Technology, Inc. Palo Alto). CA

O'KeEFE, R. M., 1985, Expert systems and operations research-mutual benefits, Journal of Operational Research Society, 36 , $125-129$.

Sabuncuoglu, I., and HommerTzheim, D., 1989, Expert simulation systems: recent developments in flexible manufacturing systems, Computers and Industrial Engineering, 16, 575-585.

SCHILLING, E. G., 1985, The role of acceptance sampling in modern quality control, Communication Statistics Theory, MATH, 14, 2769-2783.

WaTlerman, D. A., 1986, A Guide to Expert Systems (Addison Wesley, Reading, MA)

\section{Appendix}

\section{Sample consultation}

ASASP is a knowledge base system to assist the quality control engineers/managers in selecting an appropriate attribute sampling plan among the single, double and multiple sampling plans.

It will ask you series of questions regarding the lot size, fraction defective, acceptability of certain quality level by a user and other characteristics, and recommends the best plan which seems to fit well to a particular application.

Does the user desire explanations for the sampling plans? " no

Is the acceptance number of defective zero? " no

Is the sampling done under the conveyorized conditions? " no 
Does the user desire explanation about the inspection cost and elements?

"yes

*** Explanation for inspection cost and its elements ***

One important factor which affects the selection of sampling plan is the inspection cost. It basically consists of two parts: fixed cost which is proportional to the number of samples taken, and variable cost, which is proportional to the average sample size.

There are several methods and tables to calculate these parameters. Input data for each of these methods consists of producer's risk, consumer's risk, acceptable quality level (AQL), and lot tolerance proportion defective (LTPD). Sampling plans provide the acceptance criterion and required sample size.

Knowing these two parameters, acceptance criterion and sample size, the probability of acceptance and average sample size can be calculated.

The ASASP is capable of calculating average number of sample, average sample size, and consequently the inspection cost. Therefore, the value of these parameters should be determined by the users, and response given to the following questions:

One of the by-products of an acceptance sampling plan is the quantitative information about the lot quantity and process average.

In this respect, will the result of sampling be used to estimate lot quality and process average?

"yes

Two factors have a significant role in selection of double or multiple sampling plans over single sampling plan. First, the lots with marginal quality levels are given extra chance(s) for acceptance. Second, no lot is rejected due to a single defective. In addition, for a large lot size, there is often a strong objection by the producers for rejection of entire lot based on one sample. In this respect, respond to the following questions:

What do you think about the acceptability of single sampling plan by the producer?
1. least favourable
2. satisfactory
3. average
4. above average
5. most favourable

" satisfactory

What do you think about the acceptability of double sampling plan by the producer?
1. least favourable
2. satisfactory
3. average
4. above average
5. most favourable

" above average

What do you think about the acceptability of multiple sampling plan by the producer?
1. least favourable

2. satisfactory

3. average

4. above average

5. most favourable

" most favourable

In contrast to the single sampling plan, the double sampling plans have some disadvantages. They are more difficult to learn, and require more record keeping. In addition, variability in inspection load introduces extra difficulty into scheduling. As a result, double and multiple sampling plans are more subject to inspection error than single sampling plans. Considering these factors, respond to the following questions:

What is the application difficulties of single sampling plan?

1. low

2. moderate

3. high

" low

What is the application difficulties of double sampling plan?

1. low

2. moderate

3. high

" low

What is the application difficulties of multiple sampling plan?
1. low
2. moderate
3. high

" moderate

Up to now, during the consultation, you have responded to each of the individual factors which might have your selection. In general how do you rate the importance of applying appropriate sampling plan

1. least important

2. below average

3. average

4. above average

5. very important

" above average

How important are the difficulties associated with the sampling plan?

1. least important

2. below average

3. average

4. above average

5. very important

》) average 
How important is to estimate a lot quality from a sampling plan
1. least important
2. below average
3. above average
5. very important

" very important

\section{*** ASASP Recommends: ***}

single sampling plan

Do you want to continue to another consultation?

" no 